\title{
Molecular Determination of Toxigenic Potential of Fusarium spp. Isolated from Seeds of Wheat (Triticum aestivum) Genotypes and Evaluation of Levels of Fumonisins in the Grains at Harvest in Three Major Wheat Producing Counties in Kenya
}

\author{
Phanice Kheseli Otieno $\mathbb{D D}^{1}$ Susan S. Imbahale $\mathbb{D}^{1},{ }^{1}$ Vitalis Wafula Wekesa $\mathbb{D D}^{2}$ \\ Miriam Otipa, ${ }^{3}$ and Sheila Okoth $\mathbb{1}^{4}$ \\ ${ }^{1}$ Department of Applied and Technical Biology, Technical University of Kenya, P.O. Box 52428-00200, Nairobi, Kenya \\ ${ }^{2}$ Dudutech IPM Limited, P.O. Box 1927-20117, Naivasha, Kenya \\ ${ }^{3}$ Kenya Agricultural Research and Livestock Organization, Kabete, P.O. Box 1473300800, Nairobi, Kenya \\ ${ }^{4}$ School of Biological Sciences, University of Nairobi, P. O. Box 30197-00100, Nairobi, Kenya
}

Correspondence should be addressed to Phanice Kheseli Otieno; phanicekheseli@yahoo.com

Received 4 August 2021; Revised 1 December 2021; Accepted 13 December 2021; Published 15 February 2022

Academic Editor: Mehdi Rahimi

Copyright $(2022$ Phanice Kheseli Otieno et al. This is an open access article distributed under the Creative Commons Attribution License, which permits unrestricted use, distribution, and reproduction in any medium, provided the original work is properly cited.

\begin{abstract}
Infestation of cereals such as wheat by pathogenic Fusarium species (Fusarium spp.) is associated with over $25 \%$ weight loss of the grain and accumulation of hazardous mycotoxins. Potential management strategies against the pathogens include the development of more resistant wheat genotypes and the development of rapid and accurate Fusarium spp. identification methods, among other control measures. This study evaluated the toxigenic potential in populations of Fusarium spp. isolated from seeds of locally developed wheat genotypes and levels of fumonisins in the grains at harvest in three counties in Kenya. The sampling of the wheat grains took place between September 2016 and October 2017. Determination of toxigenic potential was PCR based using Tri13F/Tri13DONR and FUM1F/FUM1R specific primer pairs while detection of fumonisin levels was done using Total Fumonisin Assay 0.25/5.0 ELISA kit. Data were analyzed using ANOVA and the Tukey HSD test. FUM1 gene was detected in $60 \%$ of the Fusarium spp. analyzed. The distribution of the gene within the isolates across the three regions was as follows: Narok: $54 \%$, Uasin Gishu: 25\%, and Nakuru: 21\%. Tri13DON gene was not detected in the assessed potential deoxynivalenol (DON) producers. Fumonisin levels in the wheat samples from the three counties were significantly different $(p<0.001)$. The highest fumonisin levels, $9.6 \mathrm{ppm}$, occurred in $30.7 \%$ of the grains of the studied wheat genotypes. Relatively high fumonisin levels occurred in Njoro II, Eagle 10, Robin, and Korongo wheat genotypes with no significant difference at 0.05 confidence interval. In conclusion, the toxigenic potential amongst the Fusarium spp. studied was confirmed based on the occurrence of FUM1 gene and the detection of fumonisins in $76 \%$ of the sampled wheat grains. More research is recommended to ascertain the prevalence of the genes determining the production of DON and the other trichothecenes in Fusarium spp. prevalent in the developed wheat genotypes in the different agroecological regions in Kenya. In addition, the assessment of the occurrence and levels of the respectful mycotoxins also needs further research. These would provide additional information for the improvement of strategies put in place to manage the effects of the pathogenic Fusarium spp. in the crop and to ensure mycotoxin safety of the wheat food chain for both livestock and human consumption. It also shows the need for the development of more disease-resistant wheat varieties by wheat seedproducing companies.
\end{abstract}




\section{Introduction}

The negative effect of pathogenic Fusarium spp. in agriculture is due to the secondary metabolites (mycotoxins) they produce [1]. The toxigenic properties of mycotoxins are wide and vary in their effects ranging from phytotoxic to zootoxic activities. While accumulating in target tissues and organs of vegetables, cereals, and other organisms, mycotoxins become the cause of chronic multiorgan life-threatening diseases or may severely impair biological systems in humans and animals [2]. They are the major contaminants of fungal origin in cereal crops such as wheat and their products. The most frequently detected Fusarium spp. contaminants in wheat are DON, fumonisins (Fs), and Zearalenones [3-5]. Deoxynivalenol is a mycotoxin in the category of trichothecenes. It is produced by certain pathogenic Fusarium spp. infecting cereal crops in the field and in stores depending on the storage conditions. Fusarium graminearum is the dominant producer of DON [6]. However, F. culmorum and F. poae are among the other potential producers of DON [7]. Deoxynivalenol affects human beings and animals causing acute temporary nausea, vomiting, diarrhea, abdominal pain, headache, dizziness, and fever [8]. Other DON's effects in animals are characterized by feed refusal and reduced weight gain $[9,10]$. Fumonisins are another category of toxigenic compounds produced by certain pathogenic Fusarium spp. such as $F$. verticillioides, F. proliferatum, and F. oxysporum. The toxins have been reported to cause diverse diseases in animals such as pigs and rats and have been implicated in the etiology of human esophageal cancer [11] and WHO [12].

Fusarium spp. exhibit diversity not only in morphology but also in physiological characteristics that adapt it to a wide range of different biotic and abiotic environments. The control and management of Fusarium spp. phytopathogens has proven difficult due to their high variability and broad host range [13]. Hence, they contribute to enormous economic losses globally. A report by the Food and Agriculture Organization (FAO) of the United Nations estimated that each year about $25 \%$ of the world's food crops are contaminated with mycotoxins, causing around one billion metric tons of losses of food products [14, 15]. Consequently, due to mycotoxin-related risks associated with the consumption of cereals and their negative impact on the quality of food and feeds, countries have developed measures such as the maximum or recommended levels of the specific types of mycotoxins for food and animal feeds. For example, the Internationally Codex Alimentarius Commission (CAC) established by the World Health Organization (WHO) and FAO, in CAC/RCP, 5/2003 [16, 17], has included Fusarium toxins levels in cereals to ensure food safety.

Many mycotoxins such as fumonisins and trichothecenes are heat stable and cannot be deactivated by cooking. Hence, the only way to avoid their effect is by preventing or reducing their production in the field [2]. Accurate identification of the specific pathogens and determination of their occurrences in susceptible crops provide vital background information on the prevalence of the pathogens in the crop. However, regular assessment of the frequency of the mycotoxin biosynthetic pathway regulatory genes in populations of potential pathogenic Fusarium spp. and detection of levels of corresponding mycotoxins are equally important information. This is not only for evaluating the grain quality for consumption as seeds, feeds, and foodstuffs but also for the advancement of control strategies for ensuring the safety of wheat-based food and feeds. This is even very important with the current unpredictable climatic changes and the existence of cryptic populations. In addition, the interactions between environmental stress factors such as water activity and temperature tend to influence growth, expression of biosynthetic regulatory genes, and mycotoxin production by toxigenic fungal species $[2,18]$.

In Kenya, disease resistance and tolerance is the major driving goal of the Kenya Agricultural and Livestock Research Organization's (KALRO's) wheat research program. Although diseases remain a challenge in wheat crop production [19], some of the developed wheat varieties show some level of resistance to common wheat diseases such as wheat rust depending on the season and the location of wheat cultivation in the country [19]. For example, the Kenya Kingbird wheat genotypes have been identified as resistant to both stem and yellow rust and therefore a popular parental line in breeding for rust diseases [19]. It is appropriate that other than the cultural and chemical interventions highly recommended to reap the full potential of such genotypes, their resistance to Fusarium spp. in the field should be routinely surveyed within the prevailing and unpredictable biotic and abiotic changes. It is also of essence to constantly ascertain the toxigenic potential of the prevalent Fusarium spp. in the locally developed wheat genotypes for different agroecological regions of cultivation.

Previous research in Kenya has shown occurrences of Fusarium spp. in wheat [20-23]. Concerning the various physical, chemical, and biological approaches developed to control mycotoxin contamination in cereals, it is clear that no single control method can completely prevent the problem. Hence, systematic studies are desperately required [24-26] including frequent determination of the toxigenic potential of the persistent disease causative agents and levels of their chemical contaminants in wheat-based products. Toxigenic potential can be determined in various ways including detection of particular genes involved in the biosynthetic pathway of mycotoxins (Molecular Biology Techniques-Polymerase Chain Reactions-PCR) using the specific primers for the respective genes, followed by toxicity confirmation by evaluation of the presence and levels of the respective mycotoxins produced [5, 27, 28]. Information on the prevalence of mycotoxin biosynthetic pathway genes in the pathogenic Fusarium spp. and the level of respective mycotoxins is vital in the development or improvement of disease-resistant wheat genotypes with subsequent reduction in the production of mycotoxins in the field. It is in this respect that the study carried out a molecular assessment of the toxigenic potential in populations of Fusarium spp. isolated from seeds of locally developed wheat genotypes and evaluated the levels of mycotoxins encoded for by the detected genes in the wheat grains at harvest. 


\section{Material and Methods}

2.1. PCR Assessment of the Toxigenic Potential of the Fusarium spp. Isolates. Fusarium spp. isolated from grains of varieties of wheat genotypes were cultured on PDA for seven days and used for DNA extraction. Mycelia were scraped into Eppendorf tubes with a sterile spatula and ground with $2 \mathrm{~mm}$ wolfram beads using RetschMM 400 Mixer Mill $(30 \mathrm{~Hz}, 3 \mathrm{~min})$. Total DNA was extracted from the mycelium of each isolate (about $100 \mathrm{mg}$ wet weight) using ZR Fungal/Bacterial DNA Miniprep extraction kit (ZYMO Research, USA) and according to the manufacturer's instructions. The quality and quantity of the DNA obtained were determined using NanoDrop spectrophotometer (Thermo Scientific, USA). Fusarium spp. identification to determine the potential producers of mycotoxins among the isolates was based on internal transcribed spacer (ITS) region of the rDNA amplified by PCR using the described universal primers ITS1 (5'- TCCGTAGGTGGAACC TGCGG-3') and ITS4 (5 -TCCTCCGCTTATTGATATGC3') [29]. The ITS regions targeted for amplification were between the small nuclear $18 \mathrm{~S}$ rDNA and large nuclear $28 \mathrm{~S}$ rDNA, including 5.8S rDNA. Amplification was performed on a Thermal Cycler (Applied Biosystems 9700) with $25 \mu \mathrm{l}$ reaction mixtures containing: premix of $2 \times 12.5 \mu \mathrm{l} ; 9.5 \mu \mathrm{l}$ of nuclease-free water; $10 \mathrm{mM}$ of primer (each of ITS-1 and ITS-4); and $60 \mathrm{ng}-100 \mathrm{ng}$ genomic DNA. The amplification cycle consisted of an initial denaturation at $94^{\circ} \mathrm{C}$ for $3 \mathrm{~min}$, followed by 35 cycles at $94^{\circ} \mathrm{C}$ for $30 \mathrm{~s}, 56^{\circ} \mathrm{C}$ for $1 \mathrm{~min}, 72^{\circ} \mathrm{C}$ for $2 \mathrm{~min}$, and a final extension at $72^{\circ} \mathrm{C}$ for $8 \mathrm{~min}$. Amplified PCR products were separated on an agarose gel $(1.5 \% \mathrm{w} / \mathrm{v})$ in $1 \mathrm{X}$ TAE buffer at $70 \mathrm{~V}$ for $50 \mathrm{~min}$. The DNA amplicons were designated at $500 \mathrm{bp}$ to $550 \mathrm{bp}$ against a DNA ladder of $1 \mathrm{~kb}[29]$.

The genetic potential of Fusarium spp. isolates to produce fumonisins and trichothecenes was determined by the PCR-based molecular analysis using Tri13F/Tri13DONR [30] and FUM1F/FUM1R [31] specific primer pairs (Table 1). The pairs target the respective mycotoxin-synthesis pathway genes, Tri13DON and FUM1, for deoxynivalenol and fumonisin production, respectively. The PCR amplification for determination of the toxigenic potential of the Fusarium spp. isolates was carried out in the Veriti Thermal Cycler (Applied Biosystems, USA) according to the following program: $94^{\circ} \mathrm{C}$ for $3 \mathrm{~min}, 94^{\circ} \mathrm{C}$ for $45 \mathrm{sec}, 58^{\circ} \mathrm{C}$ for $45 \mathrm{sec}, 72^{\circ} \mathrm{C}$ for $1 \mathrm{~min}$ and 35 cycles, and final extension at $72^{\circ} \mathrm{C}$ for $5 \mathrm{~min}$ as described by $[32,33]$. The PCR products were visualized by $1 \times \mathrm{TBE}$ electrophoresis in ethidiumbromide-stained $1 \%$ agarose gel.

\subsection{Fumonisins Analysis}

2.2.1. Sample Preparation and Extraction of Fumonisins. Fifty-four (54) composite samples constituting 13 varieties of wheat genotypes were used in the analysis. Sampling of the wheat grains took place between September 2016 and October 2017 in three major wheat producing counties (Narok, Uasin Gishu, and Nakuru) in Kenya. Every representative sample was ground using a Romer series II Mill to $95 \%$ flour that could pass through a 20 -mesh sieve (sieve opening $0.84 \mathrm{~mm}$ ) and $20 \mathrm{~g}$ weighed into a clean jar. To each sample in a jar, $100 \mathrm{ml}$ of 70/30 (v/v) methanol/ water extraction solution was added. The jars were then tightly sealed and subjected to shaking at $250 \mathrm{rpm}$ for $3 \mathrm{~min}$ in an orbital shaker. The samples were extracted in a ratio of 1:5 (w: v) of sample to extraction solution, respectively. Each sample was allowed to settle, the top layer filtered through a Whatman 1 filter paper, and the filtrate diluted at the ratio of $1: 20$ with deionized water in readiness for detection of total fumonisins.

\subsubsection{Fumonisins Detection Assay and Analysis.} AgraQuant ${ }^{\circledast}$ Total Fumonisins Assay 0.25/5.0 ELISA kit, Romer Labs Singapore Pte. Ltd., was used to conduct the assay and according to the manufacturer's instructions. An appropriate number of green-bordered dilution strips were placed in a microwell strip holder. Five dilution wells were used for standards (i.e., $0,0.25,1.0,2.5$, and $5.0 \mathrm{ppm}$ ). An equal number of antibody-coated microwell strips were placed in a microwell strip holder. The required amount of conjugate $(200 \mu \mathrm{l})$ was dispensed into each green-bordered dilution well. Using a single channel pipette, $100 \mu \mathrm{l}$ of each standard/sample was added into the appropriate dilution well containing $200 \mu \mathrm{l}$ of the conjugate, using a fresh pipette tip for each standard or sample. Using an 8-channel pipette with fresh tips for each 8-well strip, the contents of each well were mixed by carefully pipetting it up and down 3 times and $100 \mu \mathrm{l}$ of the content was immediately transferred from each dilution well into a corresponding antibody-coated microwell. This was followed by incubation at room temperature for $10 \mathrm{~min}$. Contents of the microwell strips were then emptied into a waste container and the microwells were washed by filling each well with deionized water and then dumping the water from the microwell strips five times for five washes. Residue water from the wells was expelled by taping the microwell strips on several layers of absorbent paper towels on a flat surface and microwell tapped after the fifth wash. The bottom of the microwells was then dried with dry towels and $100 \mu$ l of the substrate dispensed into each microwell strip using an 8-channel pipette, followed by incubation at room temperature for $5 \mathrm{~min}$. Next, $100 \mu \mathrm{l}$ of stop solution was pipetted into each microwell strip using an 8-channel pipette and the strips read with a microwell reader using a $450 \mathrm{~nm}$ filter with a differential filter of $630 \mathrm{~nm}$. Optical density (OD) readings for each microwell were then recorded and the values were expressed as a percentage of the OD of the zero (0) standards and a dose-response curve constructed using the five standards. Since the amount of fumonisins in each standard was known, the amounts in the samples were measured by interpolation from the constructed standard curve. Results were calculated using the Romer $^{\circledast} \log /$ logit spreadsheet and log/logit regression model used for results interpretation. The correlation coefficient $(r)$ of the calibration curve was between -0.99 and -1.00 . The limit of total fumonisins detection was $0.2 \mathrm{ppm}$ while the limit of quantification was $0.25 \mathrm{ppm}$. Overall comparison of 
TABLE 1: The list of primers used to detect the potential of the Fusarium spp. isolated from grains of wheat genotypes to produce DON and fumonisins.

\begin{tabular}{lrrr}
\hline Primers used & \multicolumn{1}{c}{ ' - 5'sequences } & Product length (bp) & References \\
\hline FUM1F & CCATCACAGTGGGACACAGT & 183 & \\
FUM1R & CGTATCGTCAGCATGATGTA & & \\
TRI13F & CATCATGAGACTTGTKCRAGTTTGGG & 282 & {$[30]$} \\
TRI13DONR & GCTAGATCGATTGTTGCATTGAG & & \\
\hline
\end{tabular}

total fumonisin levels in all the wheat genotypes among the three counties was done using one-way ANOVA and Tukey HSD test to identify the differences detected. Njoro II wheat genotype was sampled in all the three counties, while Eagle10, Robin, and Kwale genotypes were obtained from only two of the sampled counties. The other wheat genotypes were available for sampling in only one county and hence no intercounty comparison was made. Fumonisin levels in Njoro II wheat genotype were therefore compared among Narok, Uasin Gishu, and Nakuru Counties while fumonisin levels in Eagle10, Robin, and Kwale were compared between Narok and Nakuru Counties using Independent Sample Ttest to detect significant differences between the two counties and between the varieties of wheat genotypes.

\section{Results}

3.1. Toxigenic Potential of Fusarium spp. Isolated from Wheat Genotypes Sampled in Three Major Wheat Producing Counties in Kenya. Both morphological characteristics (Figures 1(a) and 1(b)) and molecular analysis of the fungal isolates confirmed their identity as Fusarium spp. Result for PCR amplification of DNA of the isolates using conserved ribosomal ITS (internal transcribed spacer) regions, including the 5.8S rDNA gene exhibited a single clear amplified band that corresponded to fragment total size of between $500 \mathrm{bp}$ and $550 \mathrm{bp}$ (Figure 2). Analysis of the obtained DNA sequences and comparison with sequences from the National Centre for Biotechnology Informatics (NCBI) database using Basic Alignment Search Tool for Nucleotide Sequences (BLASTN), https://blast.ncbi.nlm.nih.gov/Blast.cgito further confirmed the species identities (Table 2). All the isolates had over $99 \%$ identity matrix value with those used for identity reference from the NCBI. Molecular analyses of the toxigenic potential of the Fusarium spp. isolates based on amplification of fum 1 gene resulted in positive identification (Figure 3) of 24 potential fumonisin producers (54\% from Narok County, 25\% from Uasin Gishu County, and 21\% from Nakuru County). Analysis of toxigenic potential based on detection of Tri13DON biosynthesis pathway gene resulted in a negative outcome in which there was no amplification of the expected 282 bp (Figure 3) for any of the Fusarium spp. isolates (Table 2) examined.

3.2. Fumonisin Levels in Grains of the Sampled Wheat Genotypes at Harvest. Chemical detection of total fumonisins in the grain samples of the wheat genotypes studied exhibited both detectable and undetectable levels of the toxins. Comparison of total fumonisin levels in all the wheat varieties sampled in the three counties (Narok, Uasin Gishu, and Nakuru) resulted in $p$ value $<0.001$. Therefore, irrespective of the type of wheat genotype, the levels of fumonisins were significantly different at a $0.05 \%$ confidence interval among the three ecological counties investigated (Figure 4). Further analysis using the Tukey HSD test revealed that the levels of fumonisins in Narok and Nakuru were significantly different $(p=0.002)$. Fumonisin levels in Uasin Gishu and Nakuru were also different $(p=0.011)$. However, total fumonisin levels in Narok and Uasin Gishu were not significantly different $(p=0.537)$. Total fumonisin levels were also significantly different $(p<0.001)$ between some of the wheat genotypes investigated (Figure 5). For example, total fumonisin levels in Njoro II and Kenya-Ibis were significantly different $(p<0.05)$, while total fumonisin levels between Njoro II and Eagle10, Robin and Korongo were not different $(p>0.05)$. Kenya Wren, Kenya Tai, and Kingbird wheat genotypes yielded either very minimal or undetectable amounts of fumonisins (Figure 6). Intercounty analysis of total fumonisin levels with respect to certain wheat genotypes (Njoro II, Eagle10, Robin, and Kwale) showed that fumonisin levels in the Njoro II wheat genotype were significantly different $(p=0.001)$ among the three counties (Figure 5). Further analysis using the Tukey HSD test found no significant differences $(p=0.99)$ in fumonisin levels in same wheat genotypes between Narok and Uasin Gishu Counties. On the other hand, the total fumonisin levels between Nakuru and both Narok and Uasin Gishu were significantly different $(p<0.05)$. Comparison of fumonisin levels in Eagle10 between Narok and Nakuru indicated significant differences $(p<0.05)$. Similarly, the comparison of fumonisin levels in Kwale wheat genotype was also significantly different $(p<0.05)$ between Narok and Nakuru Counties. However, there was no significant difference $(p>0.05)$ in fumonisin levels in Robin wheat genotype between Narok and Nakuru Counties.

\section{Discussion}

Due to the existence of cryptic populations or intraspecific diversity, forecast of the toxigenic potential of Fusarium spp. may not necessarily depend on the accurate identification of the phytopathogenic species. The findings from this study ascertained the mycotoxigenic potential in populations of Fusarium spp. infecting varieties of wheat genotypes in three of the major wheat-producing counties in Kenya. The prevalence of the FUM1 and Tri13DON biosynthesis pathway genes determining the production of the respective mycotoxins was verified based on the results obtained from PCR reactions. FUM1 gene was the only detected gene as compared to the Tri13DON gene. Out of all the Fusarium spp. isolates screened, $60 \%$ exhibited the presence of the FUM1 gene, which was detected in F. verticillioides. Eighty- 


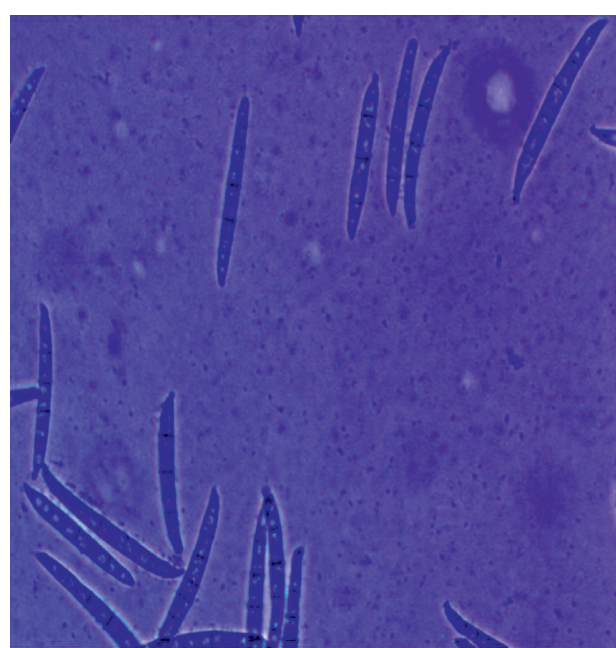

(a)

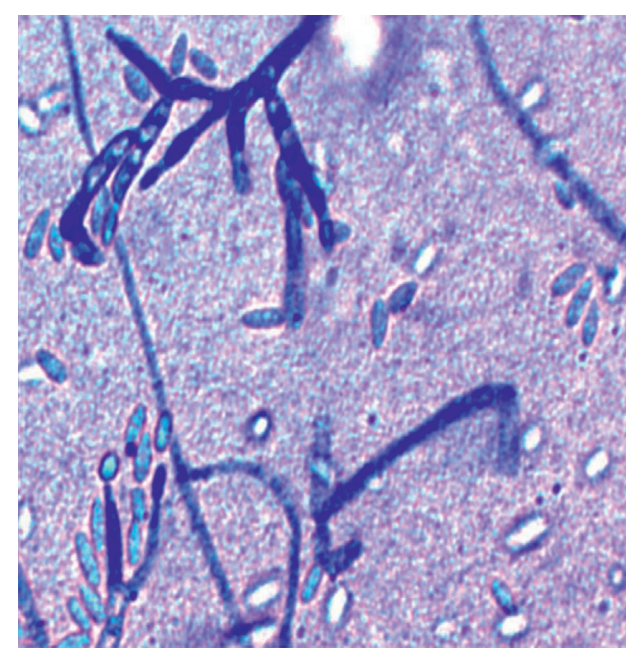

(b)

Figure 1: (a) Conidia for F. equiseti. (b) Conidia for F. verticillioides.

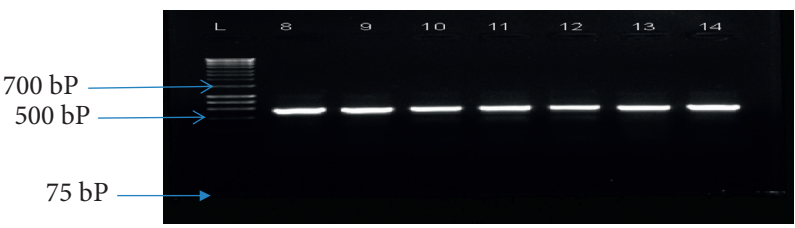

(a)

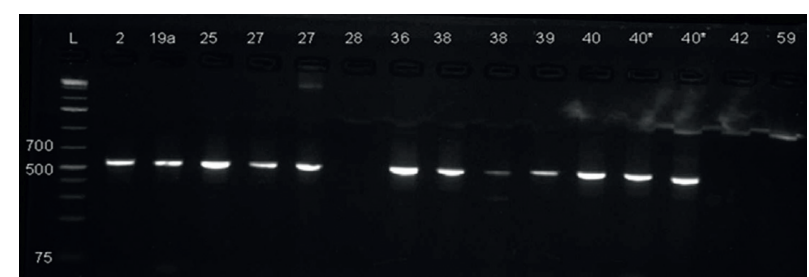

(b)

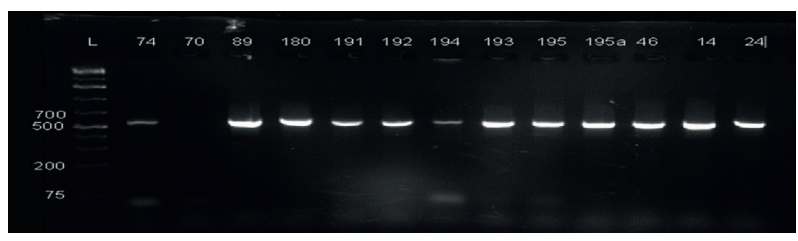

(c)

FIgURE 2: Agarose 1\% gels electrophoresis showing PCR products of the DNA for Fusarium spp. isolates obtained with the universal primers ITS1R and ITS4F. (a) Isolates' Labels: 8- PT8, 9- PT9, 10- PT10, 11- PT11, 12- PT12, 13- PT53 and 14- PT44. The ladder is 1kb. Primers used are: ITS1R and ITS4F. The band for positive result is at 500-550bp. (b) Isolates' Labels: PT2, PT19, PT25, PT27, PT28, PT36, PT38, PT39 and PT40. Primers used: ITS1 R and ITS4 F. The ladder is $1 \mathrm{~Kb}$ O Gene-ruler. Expected band is between 500-550 bp. (c) Isolates' labels: PT74, PT70, PT89, PT180, PT191, PT192, PT194, PT193, PT195, PT195a, PT46, PT14, and PT24. Primers used: ITS1 R and ITS4 F. The ladder is $1 \mathrm{~Kb} \mathrm{O}$ Gene-ruler. Expected band is between $500 \mathrm{bp}$ and $550 \mathrm{bp}$. 
TABLE 2: Mycotoxigenic potential of Fusarium spp. isolated from grains of wheat genotypes in Narok, Uasin Gishu, and Nakuru Counties in Kenya.

\begin{tabular}{lcccc}
\hline & & & & \multicolumn{2}{c}{$\begin{array}{c}\text { Mycotoxin biosynthesis pathway } \\
\text { gene examined }\end{array}$} \\
S/N & Isolate's code & Fusarium species & Region & - \\
TRI3DONR
\end{tabular}

Key: “+” gene detected, “-” gene not detected, A: Narok County, B: Uasin Gishu County, and C: Nakuru County.

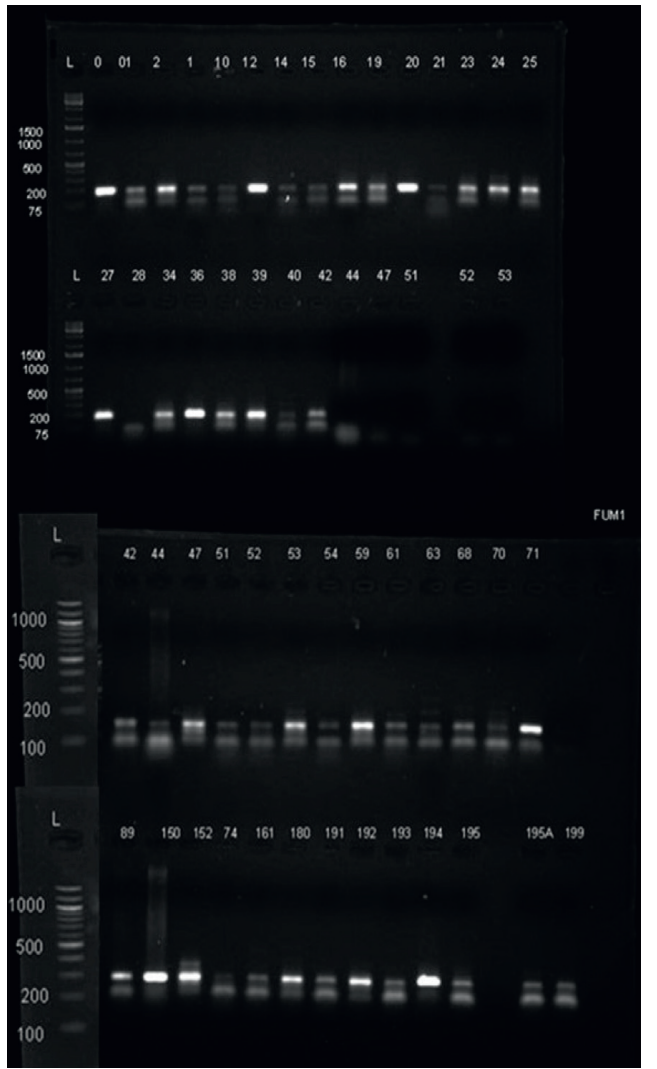

Amplification of PCR products with FUM1F and FUM1R primer pairs specific to production of fumonisins

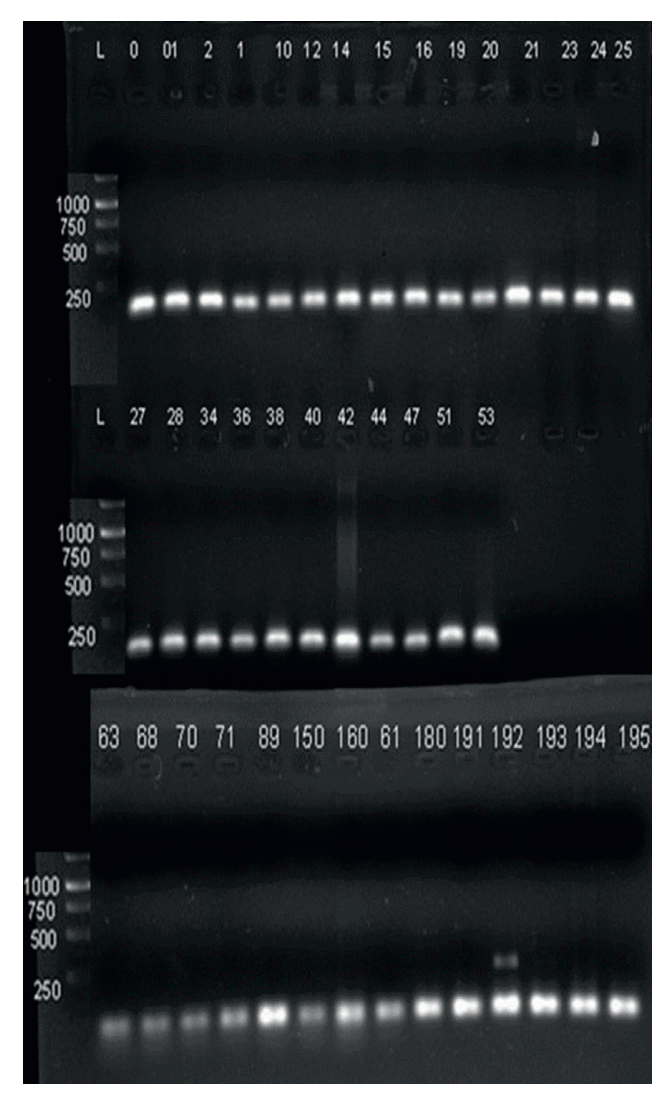

Amplification of PCR products with TRI3F/TRI3R primer pairs specific to production of deoxynivalenol.

FIGURE 3: Agarose 1\% gels electrophoresis showing PCR products for tests on toxigenic potential of Fusarium spp. isolates based on FUM1F/ FUM1R (183 bp) and Tri13F/Tri13DONR (282 bp) base pairs. (a) Amplification of PCR products with FUM1F and FUM1R primer pairs specific to production of fumonisins. (b) Amplification of PCR products with TRI3F/TRI3R primer pairs specific to production of deoxynivalenol. 


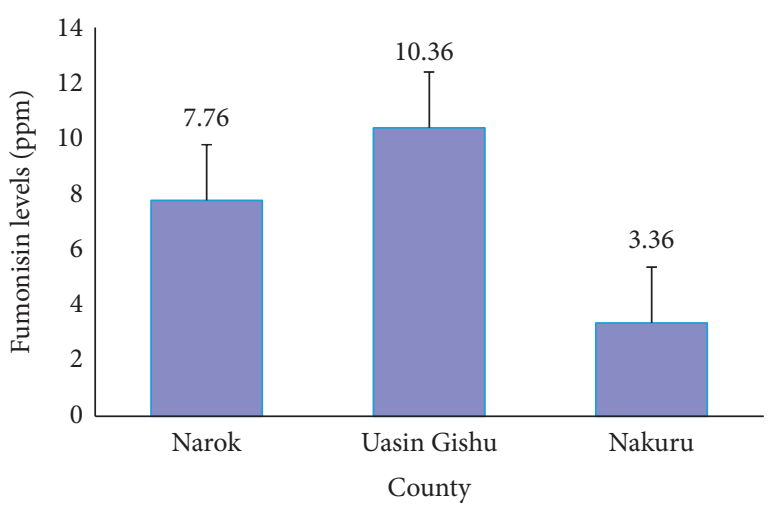

FIgURE 4: A comparison of fumonisin levels in the wheat grains sampled at harvest in three major wheat-producing Counties in Kenya.

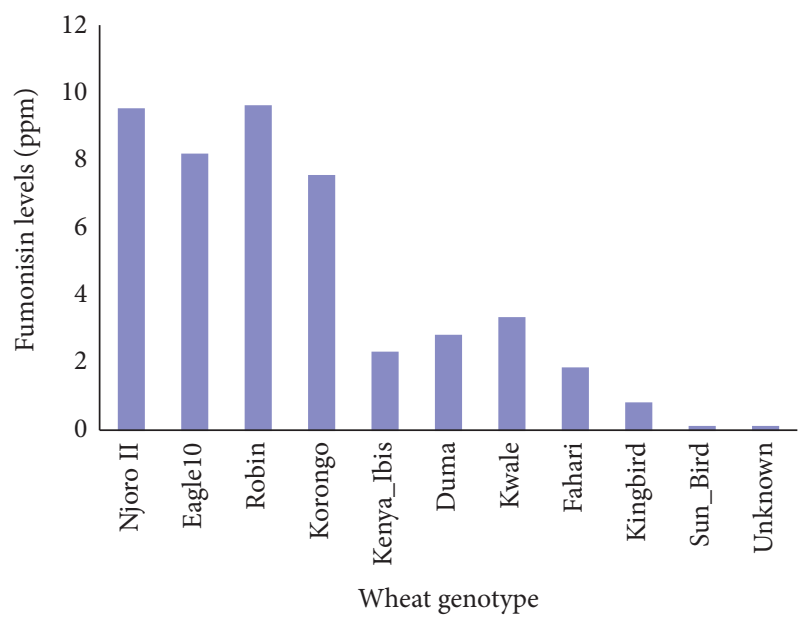

FIGURE 5: A comparison of fumonisin levels in the grains of wheat genotypes at harvest in three major wheat producing Counties in Kenya.

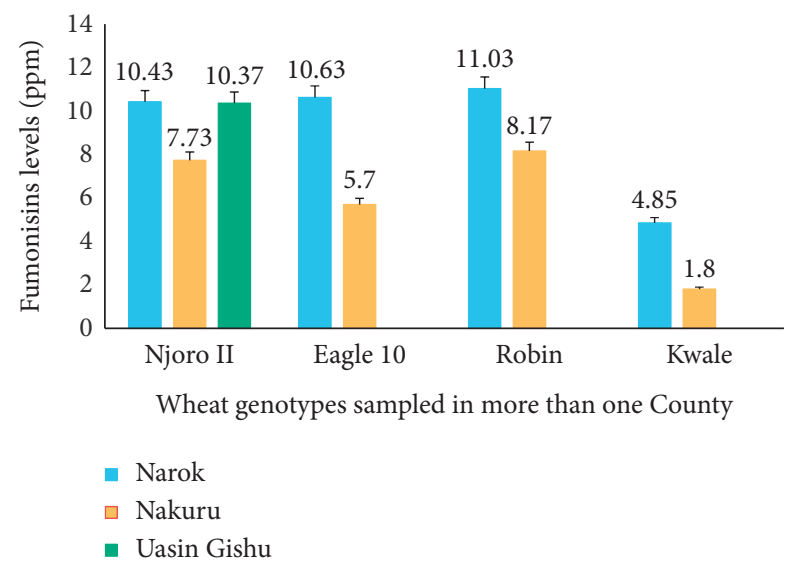

Figure 6: A comparison of fumonisin levels in the grains of Njoro II, Eagle10, Robin, and Kwale wheat genotypes in three agroecological Counties in Kenya.

nine percent (89) \% of $F$. verticillioides isolates analyzed for the presence of the FUM1 gene were positive while $11 \%$ were negative. The highest occurrence frequency of FUM1 gene was in F. verticillioides isolated from wheat genotypes sampled in Narok County.

Other potential pathogenic Fusarium spp. (F. equiseti, F. poae, Fusarium sp., F. tricinctum, and F. culmorum) were screened for the occurrence of Tri13DON, the gene determining the production of DON. However, none of them had the gene amplified. This result contradicts the findings from a similar research study by [33] who reported the amplification of the gene in F. poae. However, in the same study, the findings on F. culmorum, F. equiseti, and F. tricinctum are in agreement with our findings concerning the absence of Tri13DON gene. It is worth noting that, for $F$. poae, intraspecific differences may occur since the two research studies were done in different geographical regions. Hence, such genetic isolation may greatly influence the type of reproduction practiced resulting in genetic differences and response of the strains to ecological parameters. Our findings on the toxigenic potential of the isolates based on PCR molecular analysis support existing studies on the variation in the prevalence of the regulatory pathway genes for production of the target mycotoxins in Fusarium spp. infecting wheat crops $[5,34]$.

Fusarium spp. screened in this study are capable of producing diverse mycotoxins. However, since only FUM1 gene was detected in the assessed Fusarium spp., the respective wheat grain samples were further analyzed to determine the occurrence and levels of fumonisins at harvest. Maximum tolerable fumonisins limits can be exceeded when management strategies at the wheat production stages and weather conditions are unfavorable. Based on the above results, fumonisin levels in all the categories of wheat genotypes assessed varied from detectable to undetectable amounts. Overall, 41 (76\%) of the analyzed wheat samples were positive for detectable levels of fumonisins while 13 (24\%) of the samples had undetectable amounts. The occurrence of fumonisins in the wheat grains supports the findings by [35] in which over $68.2 \%$ of the wheat grains analyzed contained substantial levels of fumonisins partly due to the presence of $F$. verticillioides. The presence of detectable levels of fumonisins partially confirmed the results for molecular determination of the toxigenic potential of Fusarium spp. isolated from wheat grains at harvest. Although the levels of fumonisins detected in the wheat grains were, in general, lower than the acceptable maximum limit $(2000-4000 \mu \mathrm{g} / \mathrm{kg})$ in unprocessed cereal grains such as maize (Zea mays L.) grain/flour and, according to EU Commission Regulations-EC, No. 1881/2006 [36] and JECFA, 56(2001), 74(2011) (JECFA, 2017), their presence should be a precaution to food safety concerns.

Irrespective of the variety of wheat genotypes, fumonisin levels were different among the three regions targeted for study. The highest fumonisin levels occurred in wheat samples from Uasin Gishu County in which the occurrence frequency of FUM1 gene in F. verticillioides was only $25 \%$. Wheat grains samples from Nakuru County had the lowest levels of fumonisins as compared to wheat grain samples from Narok and Uasin Gishu Counties. Narok County has relatively warmer and drier conditions that favor the growth of Fusarium spp. as compared to Uasin Gishu County that is relatively cold and wet. Coupled with a higher (54\%) 
occurrence frequency of FUM1 gene in F. verticillioides isolated from wheat grains sampled in Narok County, one could have anticipated detection of higher levels of fumonisins in the grains sampled in the region. However, this was not the case, indicating that there may be other determining parameters to these findings. A common practice observed in some fields during the collection of wheat samples especially in Uasin Gishu County was the cropping of wheat and maize crops on the same farms in alternating seasons. This farming practice in essence provides conducive conditions for propagation of Fusarium spp. in susceptible crops [37-39] and hence a possible higher population of fumonisin producers in the region. Variation in quality of agroeconomic related factors ranging from farm management practices such as the quality of wheat seeds planted, fertilization, crop rotation, plowing, and hallowing, among other measures aimed at controlling pathogenic species by individual farmers may be other possible causes of the differences.

The main wheat genotype sampled in Uasin Gishu County was Njoro II. The same wheat genotype also had a higher occurrence frequency of $F$. verticillioides in the wheat grains compared to other wheat genotypes. Hence this may also account for the higher fumonisin levels detected in wheat grains sampled in Uasin Gishu County. Some of the wheat genotypes (Kenya Tai, Kingbird, Sunbird, and K. Wren) sampled exclusively in Nakuru County had very minimal or undetectable fumonisin levels while the grains of other wheat genotypes were not infected with Fusarium species. These may also have been possible reasons for the low levels of fumonisins detected in the wheat grains sampled in Nakuru County.

The occurrence of fumonisins in grains of the various wheat genotypes was mainly due to the high prevalence of $F$. verticillioides. Existing reports agree with the findings that fumonisins contamination in cereals is a worldwide concern and that $F$. verticillioides is the main producer of these toxins $[28,40,41]$. However, it is important to note that while maize was the host plant extensively studied concerning the production of fumonisins in the existing referenced work, the cereal crop targeted in our study was wheat. In Kenya, incidences of detectable fumonisin levels have been reported in the western regions of the country including some parts of Uasin Gishu [42]. In an existing study in Kenya, Kwale wheat genotype was reported to have a level of resistance to the accumulation of deoxynivalenol toxins [43]. In the current study, minimal (4.85 ppm and $1.8 \mathrm{ppm}$ ) fumonisin levels were detected in the same wheat variety in Narok and Nakuru Counties, respectively. It is also worth noting that Kenya Wren, one of the wheat genotypes developed in Kenya for resistance against wheat rust [20], did not contain detectable levels of fumonisins and neither were the respective wheat grains infected with Fusarium species.

The incidence of undetectable levels of fumonisins in grains of wheat genotypes infected with FUM1 containing Fusarium spp. might be because of varied ecological factors that affect the expression of the gene [44] and other external parameters in the regions of study. Drought stress during wheat grain development and maturation is a contributing factor to infestation by $F$. verticillioides and fumonisins production hence important in the modulation of fumonisins production $[18,45,46]$. In Kenya, the study period, 2016/2017, was characterized by prolonged drought [47] when the crop was in season. Consequently, stress related to low levels of water might have been a contributing factor to the prevalence of $F$. verticillioides and the production of the fumonisins. Fusarium verticillioides is also said to have low pathogenicity [48] but with high adaptability to hot conditions [46], which may have also accounted for the occurrence and levels of fumonisins detected in general.

In conclusion, the overall occurrence, frequency, and distribution of FUM1 and Tri13DON genes in the Fusarium spp. isolated in the three regions of study varied. The occurrence of FUM1 and detectable fumonisin levels confirmed the toxigenic potential among the Fusarium spp. isolates assessed in the study. Tri13DON gene was not detected in the Fusarium spp. isolates studied. The highest occurrence frequency of FUM1 gene was in isolates from Narok County. However, the occurrence was not directly proportional to the fumonisins levels detected in the wheat grains sampled in the same region. Uasin Gishu County had the highest overall levels of fumonisins in the grains even though there was a low (21\%) frequency of FUM1 gene in the isolates from the referenced region. Hence, more similar research work in the region is recommended to help determine the reason for these findings. There were significant variations in the levels of detectable fumonisins among the grains of the various wheat genotypes studied while some wheat genotypes had undetectable levels of fumonisins. Further research to ascertain the prevalence of genes determining the production of trichothecenes in potential producers and assessment of occurrence and levels of the respectful mycotoxins in the grains at harvest is recommended. Field tests to ascertain the resistance levels of Kenya Wren, Kenya Tai, and Sunbird wheat genotypes to pathogenic Fusarium spp. and levels of the respectful mycotoxins are also recommended. Appropriate postharvest handling of the grains is also recommended to mitigate possible harmful long-term consequences of consuming wheat grains or wheat-based products contaminated with fumonisins since over $76 \%$ of the sampled wheat grains had at least detectable levels of the toxins.

\section{Data Availability}

The data used to support these research findings are available from the corresponding author upon request.

\section{Conflicts of Interest}

The authors declare that there are no conflicts of interest concerning the publication of this paper.

\section{Authors' Contributions}

OPK and OS conceptualized and designed the study. OPK carried out field data collection and laboratory work. OPK and ISS drafted the manuscript. OS, ISS, and WVV critically reviewed the manuscript. All authors read and approved the manuscript for publication. 


\section{Acknowledgments}

The authors thank the National Commission for Science Technology and Innovation (NACOSTI) for funding this work. Much appreciation also goes to Mrs. Owiti Anne and Mr. Odongo Hesbon for assisting with technical work. The authors are indebted to Kenya Agricultural and Livestock Research Organization, Kabete, and The University of Nairobi, Chiromo Campus, which provided laboratory facilities. The research work was funded by the government of Kenya-National Commission for Science Technology and Innovation (NACOSTI) under research grant number (NACOSTI/RCD/ST\&7TH CALL/Ph.D./215).

\section{References}

[1] L. Zidan, "Morphology, pathogenicity, and molecular identification of some Fusarium species within the Gibberella fujikuroi species complex from wheat in Syria," Current Research in Environmental \& Applied Mycology, vol. 10, pp. 156-166, 2020.

[2] L. Perincherry, J. Lalak-Ká Nczugowska, and $Ł$ Stepién, "Toxins fusarium-produced mycotoxins in plant-pathogen interactions," Toxins (Basel), vol. 11, no. 11, p. 664, 2019.

[3] C. G. Lemus-Minor, M. C. Cañizares, M. D. García-Pedrajas, and E. Pérez-Artés, "Complete genome sequence of a novel dsRNA mycovirus isolated from the phytopathogenic fungus Fusarium oxysporum f. sp. dianthi," Archives of Virology, vol. 160, no. 9, pp. 2375-2379, 2015.

[4] H. I. Nirenberg, "A simplified method for identifying Fusarium spp. occurring on wheat," Canadian Journal of Botany, vol. 59, no. 9, pp. 1599-1609, 1981.

[5] S. Sadhasivam, M. Britzi, V. Zakin et al., "Rapid detection and identification of mycotoxigenic fungi and mycotoxins in stored wheat grain," Toxins, vol. 9, no. 10, pp. 1-17, 2017.

[6] M. Kushiro, "Effects of milling and cooking processes on the deoxynivalenol content in wheat," International Journal of Molecular Sciences, vol. 9, no. 11, pp. 2127-2145, 2008.

[7] M. Pasquali, M. Beyer, A. Logrieco et al., "A European database of Fusarium graminearum and F. Culmorum trichothecene genotypes," Frontiers in Microbiology, vol. 7, no. 406, 2016.

[8] P. Sobrova, V. Adam, A. VaSatkova et al., "Deoxynivalenol and its toxicity," Interdisciplinary Toxicology, vol. 3, no. 3, pp. 94-99, 2010.

[9] B. Bergsjø, W. Langseth, I. Nafstad, J. H. Jansen, and H. J. Larsen, "The effects of naturally deoxynivalenol-contaminated oats on the clinical condition, blood parameters, performance, and carcass composition of growing pigs," Veterinary Research Communications, vol. 17, no. 4, pp. 283-294, 1993.

[10] T. Goyarts and S. Dänicke, "Bioavailability of the Fusarium toxin deoxynivalenol (DON) from naturally contaminated wheat for the pig," Toxicology Letters, vol. 163, no. 3, pp. 171-182, 2006.

[11] T. J. Bucci and P. C. Howard, "Effect of fumonisin mycotoxins in animals," Journal of Toxicology: Toxin Reviews, vol. 15, no. 3, pp. 293-302, 1996.

[12] WHO (World Health Organization), Environmental Health Criteria: Fumonisins B1, World Health Organisation, Geneva, Switzerland, 2000.

[13] R. C. Ploetz, "Fusarium wilt of banana," Phytopathology, vol. 105, no. 12, pp. 1512-1521, 2015.
[14] E. Boutrif and C. Canet, "Mycotoxin prevention and control FAO programs," Revue de Medecine Veterinaire, vol. 6, pp. 681-694, 1998.

[15] M. Eskola, G. Kos, C. T. Elliott, J. Hajšlová, and R. Krska, "Worldwide contamination of food crops with mycotoxins: validity of the widely cited "FAO estimate" of $25 \%$," Critical Reviews in Food Science and Nutrition, vol. 60, pp. 2773-2789, 2020.

[16] Gap and GMP, Code of Practice for the Prevention and Reduction of Mycotoxin Contamination in Cereals 2, Good Agricultural Practices (Gap) and Good Manufacturing Practices (Gmp), San Francisco, CF, USA, 2014.

[17] Codex Alimentarius Commission, Code of Practice for the Prevention and Reduction of Arsenic Contamination in Rice, Codex Alimentarius Commission, Rome, Italy, 2017.

[18] A. Medina, M. Schmidt-Heydt, A. Rodríguez, R. Parra, R. Geisen, and N. Magan, "Impacts of environmental stress on growth, secondary metabolite biosynthetic gene clusters and metabolite production of xerotolerant/xerophilic fungi," Current Genetics, vol. 61, no. 3, pp. 325-334, 2015.

[19] J. Kamwaga, G. Macharia, L. Boyd et al., "Kenya wheat production handbook 2016," Experimental Agriculture, vol. 37, no. 1, 2016.

[20] J. W. Muthomi, J. K. Ndung'u, J. K. Gathumbi, E. W. Mutitu, and J. M. Wagacha, "The occurrence of Fusarium species and mycotoxins in Kenyan wheat," Crop Protection, vol. 27, no. 8, pp. 1215-1219, 2008.

[21] J. W. Muthomi, S. L. Musyimi, J. M. Wagacha, and R. D. Narla, "Occurrence of Fusarium species and associated T2-toxin in Kenyan wheat," Agricultural Sciences, vol. 3, no. 1, pp. 24-34, 2012.

[22] M. Wagacha, J. M. Steiner, U. Dehne et al., "Diversity in mycotoxins and fungal species infecting wheat in Nakuru district, Kenya," Journal of Phytopathology, vol. 158, no. 7-9, pp. 527-535, 2010.

[23] J. M. Wagacha, N. K. Njeru, O. O. Okumu, J. W. Muthomi, and C. K. Mutegi, "Occurrence of Fusarium head blight of wheat and associated mycotoxins in Narok and Nakuru Counties, Kenya," World Journal of Agricultural Research, vol. 4, no. 4, pp. 119-127, 2016.

[24] S. Prasad, M. Shams-Ghahfarokhi, R. Lawrence Brown et al., "Functional agents to biologically control deoxynivalenol contamination in cereal grains," Frontiers in Microbiology, vol. 7, no. 395, 2016.

[25] L. Shah, A. Ali, M. Yahya et al., "Integrated control of fusarium head blight and deoxynivalenol mycotoxin in wheat," Plant Pathology, vol. 67, no. 3, pp. 532-548, 2018.

[26] J. Wan, B. Chen, and J. Rao, "Occurrence and preventive strategies to control mycotoxins in cereal-based food," Comprehensive Reviews in Food Science and Food Safety, vol. 19, no. 3, pp. 928-953, 2020.

[27] A. M. Omori, E. Y. S. Ono, J. G. Bordini, M. T. Hirozawa, M. H. P. Fungaro, and M. A. Ono, "Detection of Fusarium verticillioides by PCR-ELISA based on FUM21 gene," Food Microbiology, vol. 73, pp. 160-167, 2018.

[28] D. A. Sampietro, P. Marín, J. Iglesias et al., "A molecularbased strategy for rapid diagnosis of toxigenic Fusarium species associated with cereal grains from Argentina," Fungal Biology, vol. 114, no. 1, pp. 74-81, 2010.

[29] D. Walker, L. Castlebury, A. Rossman, and J. White, "New molecular markers for fungal phylogenetics: two genes for species-level systematics in the Sordariomycetes (Ascomycota)," Molecular Phylogenetics and Evolution, vol. 64, pp. 500-512, 2012. 
[30] E. A. Chandler, D. R. Simpson, M. A. Thomsett, and P. Nicholson, "Development of PCR assays to Tri7 and Tri13 trichothecene biosynthetic genes, and characterisation of chemotypes of Fusarium graminearum, Fusarium culmorum and Fusarium cerealis," Physiological and Molecular Plant Pathology, vol. 62, no. 6, pp. 355-367, 2003.

[31] B. H. Bluhm, M. A. Cousin, and C. P. Woloshuk, "Multiplex real-time PCR detection of fumonisin-producing and trichothecene-producing groups of Fusarium species," Journal of Food Protection, vol. 67, no. 3, pp. 536-543, 2004.

[32] A. M. Lenart, A. Klimek-Kopyra, and P. M. Boroń, "Morphological and molecular identification and PCR amplification to determine the toxigenic potential of Fusarium spp. isolated from maize ears in southern Poland," Phytoparasitica, vol. 41, no. 3, pp. 241-248, 2013.

[33] K. Wolny-Koładka, A. Lenart-Boroñ, and P. Boroñ, "Species composition and molecular assessment of the toxigenic potential in the population of Fusarium spp. Isolated from ears of winter wheat in southern Poland," Journal of Applied Botany and Food Quality, vol. 88, pp. 139-144, 2015.

[34] H. A. El Yazeed, A. Hassan, R. E. A. Moghaieb, M. Hamed, and M. Refai, "Molecular detection of Fumonisins-producing Fusarium species in animal feeds using polymerase chain reaction (PCR)," Journal of Applied Sciences Research, vol. 7, no. 4, pp. 420-427, 2011.

[35] K. Chehri, S. T. Jahromi, K. R. N. Reddy, S. Abbasi, and B. Salleh, "Occurrence of Fusarium spp. and fumonisins in stored wheat grains marketed in Iran," Toxins, vol. 2, no. 12, pp. 2816-2823, 2010.

[36] European Commission, "Commission Recommendation of 17 August 2006 on the presence of deoxynivalenol, zearalenone, ochratoxin A, T-2, and HT-2 and fumonisins in products intended for animal feeding," Official Journal of the European Union, vol. 299, pp. 7-9, 2006.

[37] M. D. Keller, A. B. Baudoin, W. E. Thomason, and B. A. Vinatzer, The contribution of within-field inoculum sources of gibberella zeae to Fusarium head blight in winter wheat and barley, $\mathrm{PhD}$ Thesis, Virginia Polytechnic Institute, Blacksburg, VA, USA, 2011.

[38] N. K. Njeru, J. W. Muthomi, C. K. Mutegi, and J. M. Wagacha, "Effect of cropping systems on the accumulation of fusarium head blight of wheat inocula in crop residues and soils," Journal of Plant Sciences, vol. 11, no. 1, pp. 12-21, 2016.

[39] N. Njeru, M. Wagacha, J. W. Muthomi, and C. Mutegi, "Role of soil and crop residues as sources of inoculum for Fusarium head blight of wheat," in Proceedings of the Fourth RUFORUM Biennial Regional Conference, pp. 213-217, Maputo, Mozambique, January 2014.

[40] E. Cendoya, M. Nichea, M. Monge, M. Sulyok, S. M. Chiacchiera, and M. Ramirez, "Fumonisins occurrence in wheat-based products from Argentina," Food Additives and Contaminants: Part B, vol. 12, pp. 1-7, 2018.

[41] J. P. Rheeder, W. F. O. Marasas, and H. F. Vismer, "Production of fumonisins analogs by Fusarium species," Applied and Environmental Microbiology, vol. 68, no. 5, pp. 2101-2105, 2002.

[42] S. K. Mutiga, V. Hoffmann, J. W. Harvey, M. G. Milgroom, and R. J. Nelson, "Assessment of aflatoxin and fumonisin contamination of maize in western Kenya," Phytopathology, vol. 105, no. 9, pp. 1250-1261, 2015.

[43] O. Otieno and N. Njeru, "Occurrence of Fusarium head blight, associated mycotoxins, and of resistance of wheat germplasm," Agricultural Sciences, vol. 3, no. 1, 2014.
[44] N. J. Alexander, R. H. Proctor, and S. P. McCormick, "Genes, gene clusters, and biosynthesis of trichothecenes and fumonisins in Fusarium," Toxin Reviews, vol. 28, no. 2-3, pp. 198-215, 2009.

[45] D. Ferrigo, A. Raiola, and R. Causin, "Plant stress and mycotoxin accumulation in maize," Agrochimica, vol. 58, no. 1, pp. 116-127, 2014

[46] D. Ferrigo, A. Raiola, and R. Causin, "Fusarium toxins in cereals: occurrence, legislation, factors promoting the appearance and their management," Molecules, vol. 21, no. 5, 2016.

[47] W. Schmidt, A. Peter Uhe, J. Kimutai, F. Otto, and H. Cullen, The Drought in Kenya, 2016-2017 Climate and Development Knowledge Network and World Weather Attribution Initiative, Kenya Meteorological Department, Nairobi, Kenya, 2017.

[48] M. W. Parsons and G. P. Munkvold, "Effects of planting date and environmental factors on fusarium ear rot symptoms and fumonisin Blaccumulation in maize grown in six North American locations," Plant Pathology, vol. 61, no. 6, pp. 1130-1142, 2012. 